\title{
Weak Amplitude Modulated (AM) Signal Detection Algorithm for Software-Defined Radio Receivers
}

\author{
Thomas Kokumo Yesufu ${ }^{1}$, Abimbola Oyewole Atijosan ${ }^{2}$ \\ ${ }^{1}$ Department of Electronic and Electrical Engineering, Obafemi Awolowo University, Ile-Ife, Nigeria \\ ${ }^{2}$ Cooperative Information Network, Obafemi Awolowo University, Ile-Ife, Nigeria
}

Email address:

thomas_yesufu@yahoo.com (T. K. Yesufu), bimbo06wole@yahoo.com (A. O. Atijosan)

\section{To cite this article:}

Thomas Kokumo Yesufu, Abimbola Oyewole Atijosan. Weak Amplitude Modulated (AM) Signal Detection Algorithm for Software-Defined Radio Receivers. International Journal of Intelligent Information Systems. Vol. 4, No. 4, 2015, pp. 79-83. doi: 10.11648/j.ijiis.20150404.12

\begin{abstract}
In this paper a software implementation of a reconfigurable Amplitude Modulated (AM) receiver for weak AM signals detection with reduced processing latency is presented. The Stochastic Resonance (SR) algorithm, which is a technique for weak signal detection was developed for software defined, AM receiver. The performance of the SR based AM receiver was evaluated in terms of its output Signal to Noise (SNR) Ratio, and processing latency. From the results from our simulations, this approach provides better performance and lesser processing latency requirement than conventional signal processing methods for detecting AM signals.
\end{abstract}

Keywords: Weak Signal Detection, Reconfigurable Receivers, Amplitude Modulation Receivers, Signal Processing

\section{Introduction}

Traditional communication systems have typically been implemented using custom hardware solutions (Iancu et al 2004). The shift away from hard-wired communication terminals towards software defined and reconfigurable communication devices will introduce new and efficient ways for existing and future technologies to implement radio communication devices. The phrase "software radio" refers to the class of reconfigurable radios in which the physical layer behavior can be significantly altered without the change in the hardware (Shajedul Hasan et al, 2005).

Software radios are fundamentally different from hardware radios, and new algorithmic ideas are needed to make them viable (Matteo, 2002). Challenges confronting software defined AM receivers include signal degradation which militates against the effective use of the AM band (Yesufu, 1995). Other challenges stem from the poor signal to noise ratio of weak AM stations (Oliver et al., 2003), and the latency requirements of signal processing algorithms for $\mathrm{AM}$ receivers and other emerging applications (Anthony, 2012); latency can be described as the amount of time it takes the input signal to pass through the system, and reach the output, and it plays a vital role in the design of software radios.

Recovery of a signal of interest that is obscured by noise is becoming the limiting process in many applications particularly since modern data acquisition techniques make capture and processing of the final signal of interest quite straightforward (White and Williams, 2012). Developments of signal processing algorithms are needed (CSDR, 2007), to advance the frontiers of software defined AM receivers, improve performance and confront the challenges of latency and buffer sizes that arise in using Digital Signal Processing (DSP) platforms for radio detection.

\section{Theoretical Development}

Amplitude modulated signals can be represented mathematically as

$$
s(t)=A\left[1+k_{a} m(t)\right] \cos w_{c} t
$$

Where $m(t)=A \cos \omega_{m} t$ is the baseband or message signal with a bandwidth of $\mathrm{W}$ and amplitude $\mathrm{A}, \mathrm{c}(\mathrm{t})=$ $\cos \left(w_{C} t\right)$ is the carrier wave, The parameter $k_{a}$ is a positive constant called the amplitude sensitivity of the modulator.

In a coherent receiver the input AM radio signal $s(t)$ is multiplied by a copy of the carrier wave $\mathrm{c}(\mathrm{t})$ and the result is passed through a lowpass filter (LPF) with a cut-off frequency $\mathrm{W}$. The signal at the output of the multiplier can be 
represented as

$$
y(t)=s(t) * c(t)
$$

The lowpass filter completely suppresses those spectral components of signal $y(t)$. At the output of the filter, a signal proportional to the original signal $m(t)$ can be obtained. For a non-coherent detector (based on a square law device), the output of the detector can be described as

$$
y(t)=s(t) * s(t)
$$

The output of the detector is then lowpass filtered and the square root obtained to produce an output proportional to $\mathrm{m}(\mathrm{t})$ with a DC offset.

Stochastic resonance (SR) is a phenomenon in which a nonlinear system is subjected to a periodic modulated signal so weak as to be normally undetectable, but it becomes detectable due to resonance between the weak deterministic signal and stochastic noise (Weinstein, 2008).

Consider a one-dimensional nonlinear stochastic system which is defined by the nonlinear langenvin equation:

$$
\frac{\partial x(t)}{\partial t}=-V^{\prime}(x)+\xi(t)
$$

Where $x(t)$ is the state of the SR system, $\xi(t)$ represents bandlimited Gaussian white noise with zero mean. $V(x)$ is a double-well potential with positive parameters $b$ and $a$ characterizing the system. $V(x)$ is represented as:

$$
V(x)=\frac{1}{4} b x^{4}-\frac{1}{2} a x^{2}
$$

Applying $y(t)$ to (4), the equation can then be written as

$$
\frac{\partial x(t)}{\partial t}=-V^{\prime}(x)+p(t)=a x-b x^{3}+p(t)
$$

where $p(t)=y(t)+\xi(\mathrm{t})$

Equation (6) is a first order differential equation and can be approximately solved using the Runge-Kutta method.

The Runge-Kutta method for solving differential equations is a more accurate method of great practical importance. The method is well suited for computers because it needs no special starting procedure, makes light demand on storage and repeatedly uses the same straight forward computational procedure. It is also numerically stable (Kreyszig, 2006).

For $y^{\prime}=f(x, y), y\left(x_{0}\right)=y_{0}, \quad$ we generate approximations $y_{n}$ to $y\left(x_{0}+n h\right)$ for $h$ fixed and for $n=0,1,2, \ldots \ldots \ldots \ldots n-1$ using the recursion formula

$$
y_{n+1}=y_{n}+\frac{1}{6}\left(k_{1}+2 k_{2}+2 k_{3}+k_{4}\right)
$$

Where

$$
\begin{gathered}
k_{1}=h f\left(x_{n}, y_{n}\right) \\
k_{2}=h f\left(x_{n}+\frac{h}{2}, y_{n}+\frac{1}{2} k_{1}\right) \\
k_{3}=h f\left(x_{n}+\frac{h}{2}, y_{n}+\frac{1}{2} k_{2}\right)
\end{gathered}
$$

$$
k_{4}=h f\left(x_{n}+h, y_{n}+k_{3}\right)
$$

The algorithm steps can be described as follows:

$$
\begin{gathered}
x_{n+1}=x_{n}+\frac{1}{6}\left(k_{1}+2 k_{2}+2 k_{3}+k_{4}\right) \\
\mathrm{n}=0,1, \ldots \mathrm{N}-1, x(0)=x_{0} \\
k_{1}=h\left[a x_{n}-b x_{n}^{3}+p r_{n}\right] \\
k_{2}=h\left[a\left(x_{n}+\frac{k 1}{2}\right)-b\left(x_{n}+\frac{k 1}{2}\right)^{3}+p r_{n+1}\right] \\
k_{3}=h\left[a\left(x_{n}+\frac{k 2}{2}\right)-b\left(x_{n}+\frac{k 2}{2}\right)^{3}+p r_{n+1}\right. \\
k_{4}=h\left[a\left(x_{n}+k 3\right)-b\left(x_{n}+k 3\right)^{3}+p r_{n+1}\right]
\end{gathered}
$$

$x_{n}$ and $p r_{n}$ denote the nth sample of $x(t)$ and $p(t)$, respectively, $h$ denotes time step, $\mathrm{N}$ is the total number of sampling points. The developed system is as shown in Fig. 1 (Atijosan, 2010).

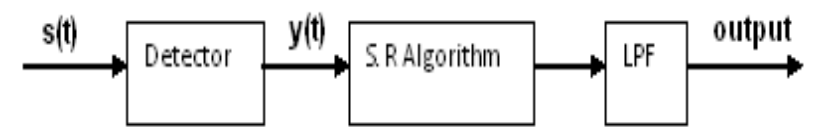

Fig. 1. Block Diagram of the Improved AM Receiver Using Stochastic Resonance (SR).

\section{Simulation}

The simulation was carried out using Matlab 7.1. The corresponding simulation examples are given to validate the effect of SR on detection of weak AM signals. A modulation index of 0.6 was used. A cosine wave with centre frequency of $20 \mathrm{~Hz}$ was used as the baseband signal, carrier and sampling frequencies were set at $1 \mathrm{kHz}$ and $10 \mathrm{kHz}$, respectively. Fig. 2 shows the baseband signal after amplitude modulation in time and frequency domain. The weak AM signal was simulated by adding additive white Gaussian noise (AWGN) signal, at various levels of signalto-noise ratio (SNR), to the AM signal at the input to the demodulator.

\section{Results and Discussion}

The performance comparison of a SR based product detector and a product detector at input SNR of $10 \mathrm{~dB}$ is shown in Fig. 3. The time domain plot shows that the SR based coherent detector performed better than the product detector. Fig. 4 compares the performance of SR based product detector, SR based square law detector, square law and product detector based on their input SNR and output SNR. 

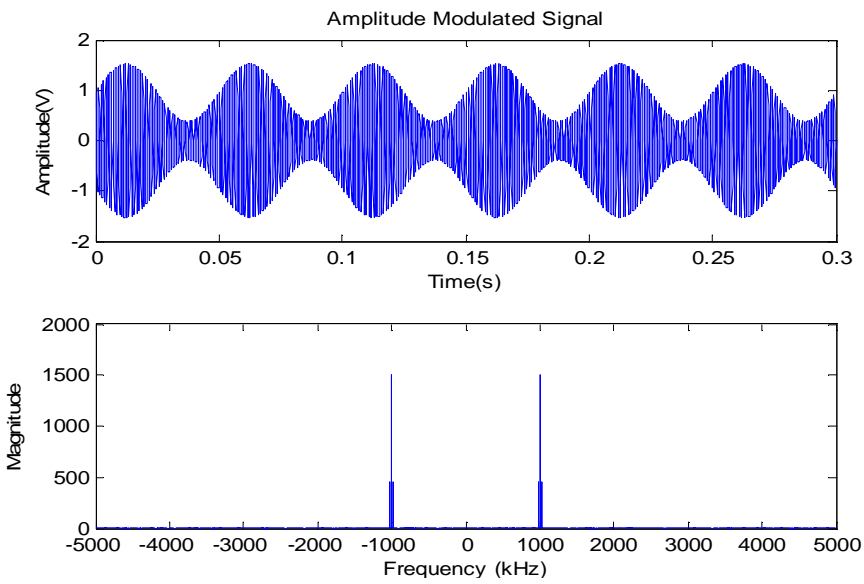

Fig. 2. (a) Baseband signal after Amplitude Modulation in time domain. (b) Baseband signal in frequency domain after Amplitude Modulation
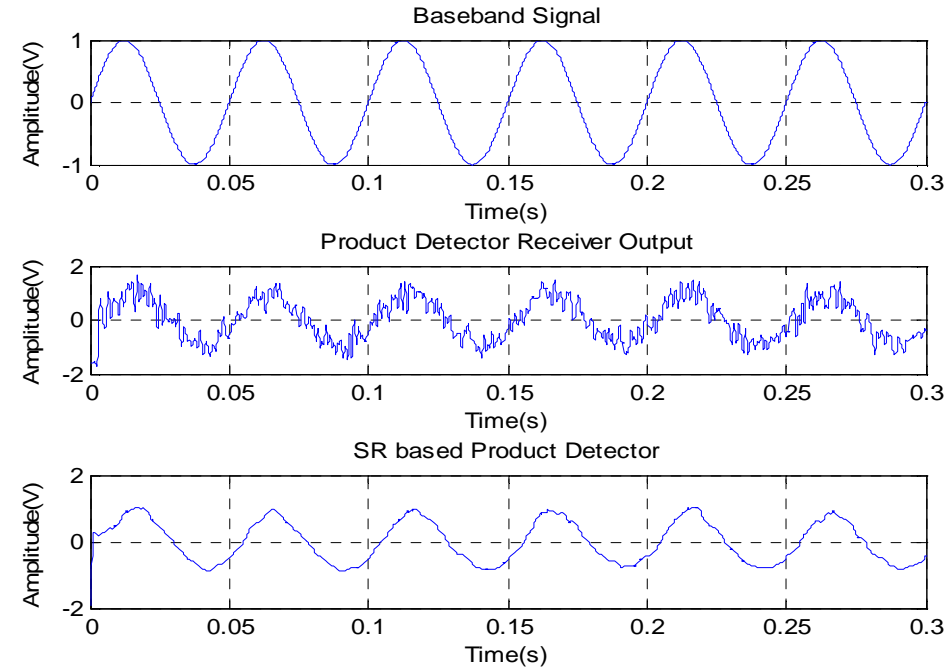

Fig. 3. Performance Comparison of SR based product detector and product detector at input SNR of 10dB.

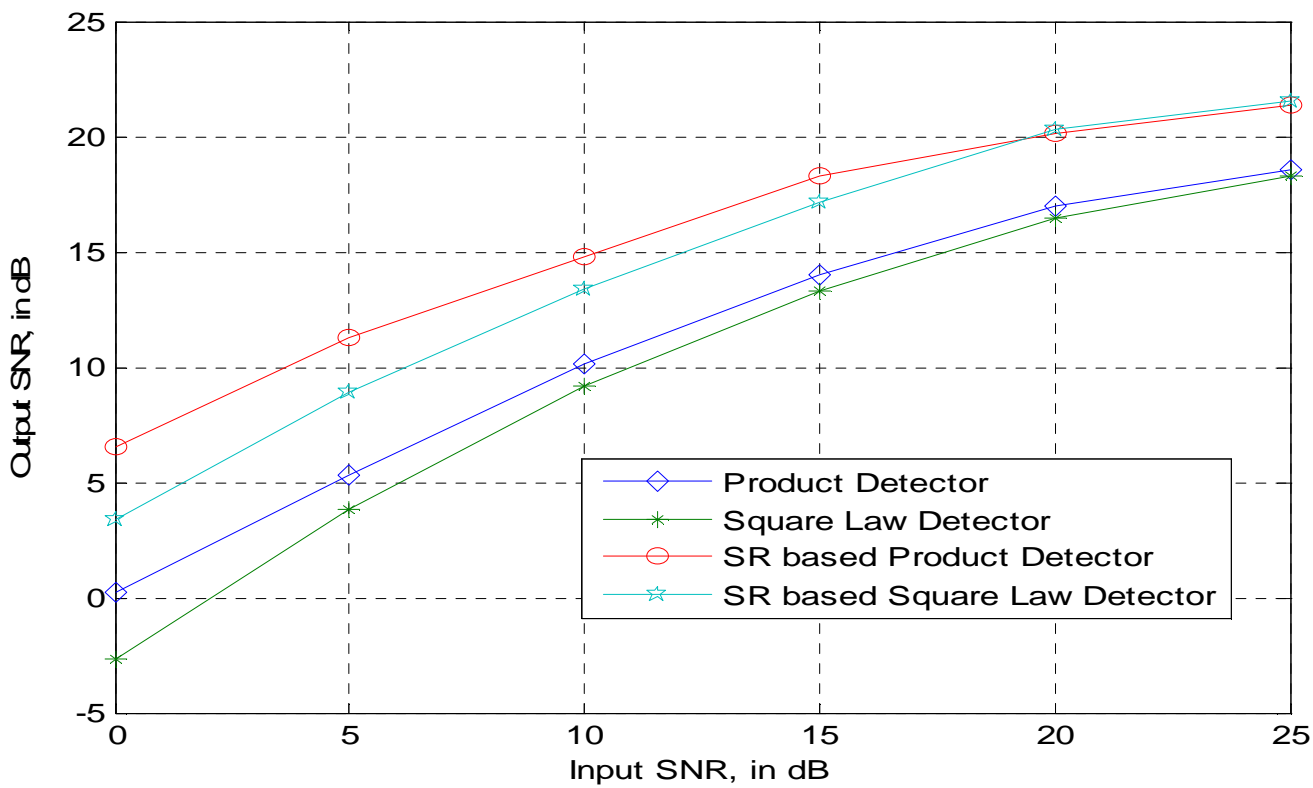

Fig. 4. Performance comparison of stochastic resonance (SR) based product detector, SR based square law detector, square law and product detector. 


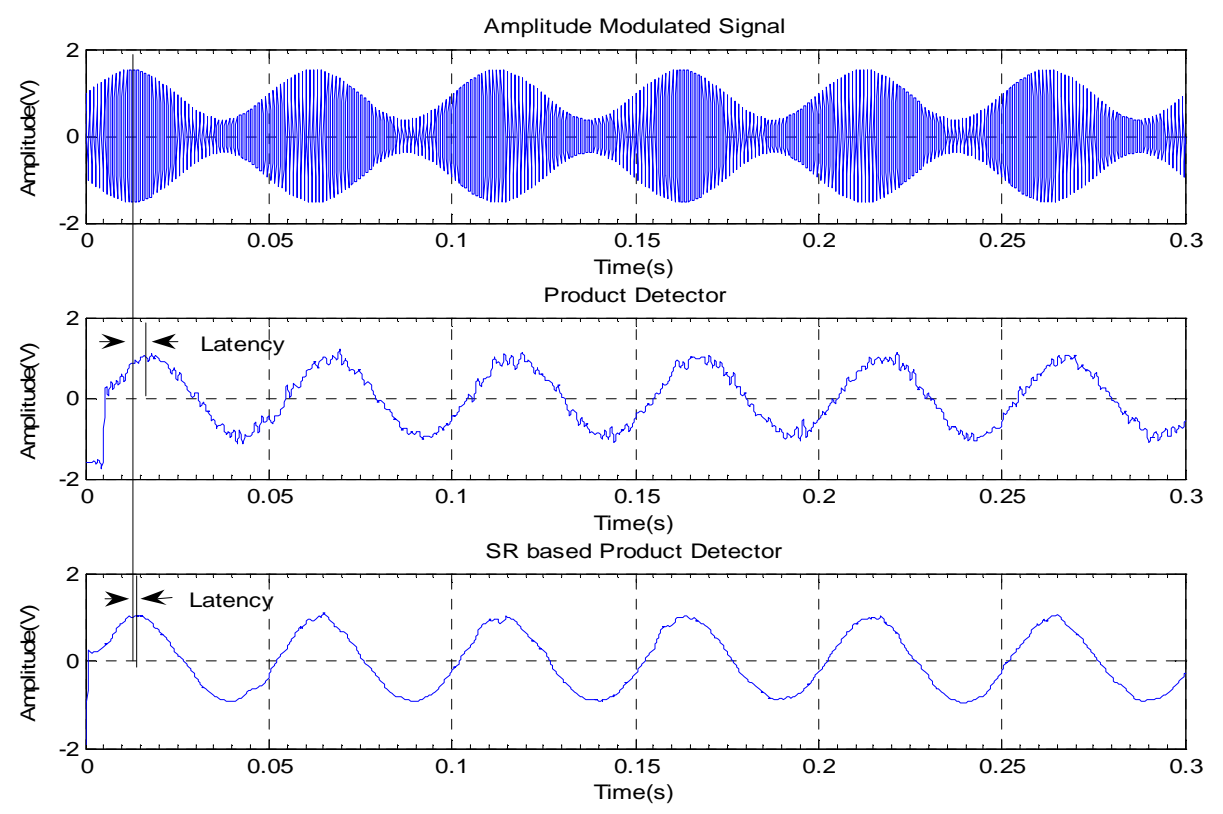

Fig. 5. Latency and Performance Comparison of a product detector (Lowpass filter length of 100) and an SR based product detector (Lowpass filter length of 10) at input SNR of $20 \mathrm{~dB}$.
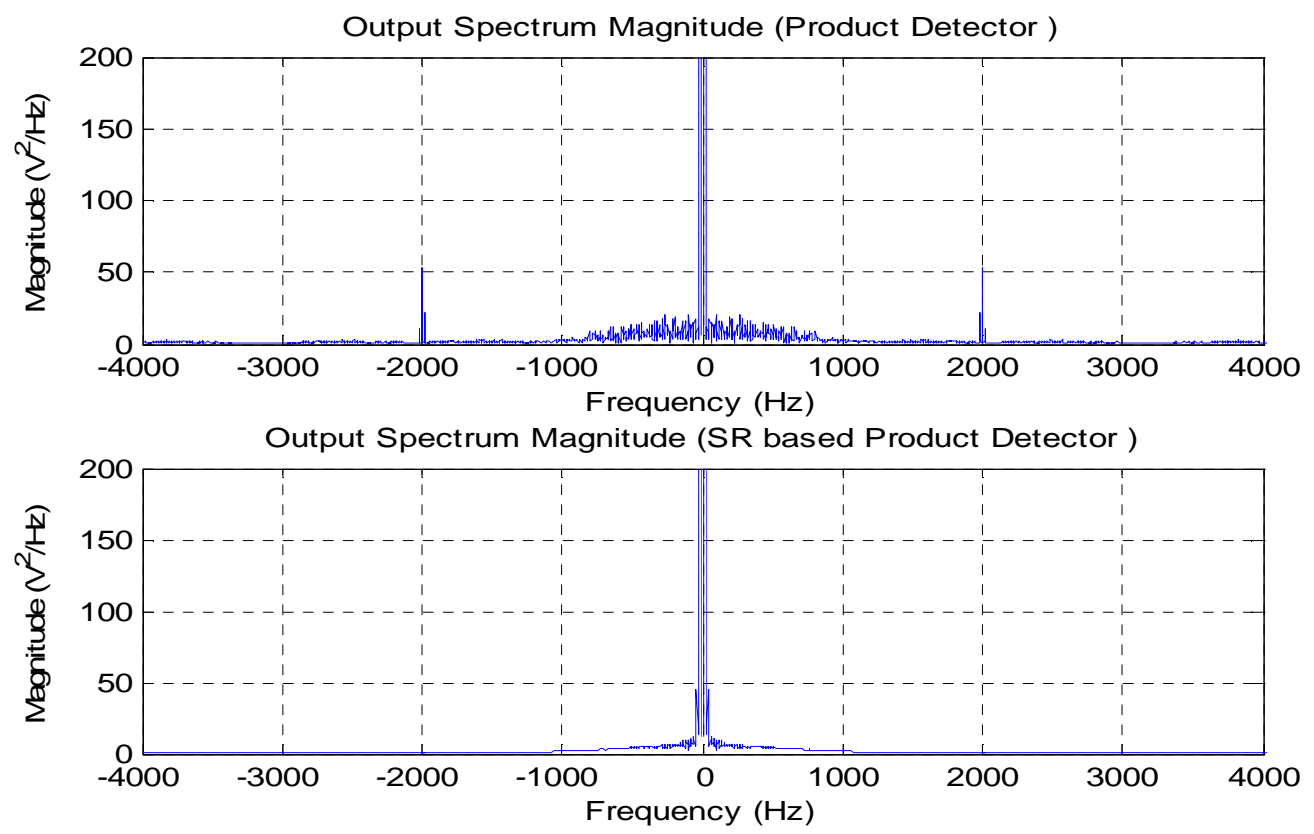

Fig. 6. Spectral comparison of product detector and SR based product detector at the same low pass filter length of 10 and input SNR of $20 \mathrm{~dB}$.

Fig. 5 shows the latency and performance comparison of a product detector, with low pass filter length of 100 and an SR based product detector with a low pass filter length of 10 , at input SNR of $20 \mathrm{~dB}$. The SR based product detector has a processing latency of $1.4 \mathrm{~ms}$ while the product detector has a processing latency of $4 \mathrm{~ms}$. Fig. 6 compares the performance of a product detector and an SR based product detector using the same low pass filter length of 10 at an input SNR of $20 \mathrm{~dB}$. The spectral plot (Fig. 6) shows that the product detector has a higher noise floor when compared with that of the SR based product detector, the output quality of the product detector can be increased by using a longer filter length but at a cost of increasing the latency time, as shown in Fig. 5.

\section{Conclusion}

In this paper, an efficient algorithm for weak signal detection in software defined AM receiver based on the stochastic resonance model for extracting weak signals was developed. From the results, this approach provides a better performance and lower latency than some conventional signal processing methods for detecting AM signals. 


\section{References}

[1] Anthony, S. (2012): Increasing Wireless Network Speed by $1000 \%$, by Replacing Packets with Algebra, Available Online: http://www.extremetech.com/ computing/138424- increasingwireless-network-speed-by-1000-by-replacing-packets-withalgebra.

[2] Atijosan, A.O. (2010): Development of a Reconfigurable Amplitude Modulation Receiver, An M.Sc. Thesis Submitted to the Department of Electronic and Electrical Engineering, Obafemi Awolowo University, Ile-Ife, Nigeria.

[3] Centre for Software Defined Radio (CSDR), (2007). "Software Defined Radio: Terms, Trends and Perspectives". Aalborg Universitet, Aalborg, Denmark. January 2007, pp. 6.

[4] Iancu, D., Glossner, J., Ye, H., Abdelila, Y., Stuart S. (2003), "Software AM Radio Implementation". Sandbridge Technologies Inc, 1 N Lexington Avennue, 10th Floor, White Plains, New York 10601.

[5] Kreyszig, E. (2006). " Advanced Engineering Mathematics ", 8th Edition. John Wiley and Sons, Inc, pp. 947 - 948.
[6] Olivier, R., Denby, B. and Sid-Ahmed, H. (2003). "A Real Time Software Radio for the AM Long Wave Band". Laboratoire des Instruments et Systemes d'Ile de France (LISI6F), Universite Pierre et Marie Curie, B.C. 252, 4 PLACE Jussieu, 75252 Paris Cedex 05, France, pp. 1-4.

[7] Matteo, F. (2002). 'Algorithmic advances for software radios', Vanu Inc, porter square, suite 18, Cambridge, MA 02140, pp.1.

[8] Shajedul Hasan S.M. and Balister P. (2005): "Prototyping a Software Defined Radio Receiver Based on USRP and OSSIE”. Chameleonic Radio Technical Memo No. 1.

[9] Weisstein, E. (2008). "Stochastic Resonance." From MathWorld - A Wolfram WEB Resource, Available Online: http://mathworld.wolfram.com/ StochasticResonance.html.

[10] White, A. and Williams, A. (2012): Systems, Methods and Computer-Readable Media for Configuring Receiver Latency, Available Online: Http://www.google.com.ng/patents/ US 8966109 B2.

[11] Yesufu, T.K. (1995). "The Dynamics of a Regenerative Receiver". Proceedings of the Regional Workshop on Radio Communication in Africa, (Radio Africa 1995) held at Obafemi Awolowo University Ile-Ife, Nigeria, pp. 185. 